titers. The 3 patients with high titers and a decrease in growth velocity all had an isolated hereditary growth hormone deficiency.

In conclusion, most patients with negative, low, or medium anti-HGH antibody titers grow adequately. The presence of high level titers inhibited the growth response to $\mathrm{HGH}$ administration.

\section{High Affinity Estrogen Receptors in Uterus of Early Mal- nourished Rats}

SANTIAGO MUZZO AND ABRAHAM ZVAIGHAFT Institute of $\mathrm{Nu}$ -
trition and Food Technology, Universidad de Chile, Santiago, Chile

We have found that marasmic malnutrition in rats permanently decreases uterine cell number. On the other hand, a decrease of total plasma estrogen levels in mothers of small for gestational age newborns has been demonstrated. We have found, also, decreased levels of sex hormone binding globulin in these mothers, suggesting lower levels of free plasma estrogens. As an index of hormone action upon the target cells, we studied uterine estradiol receptors of early malnourished rats. Malnutrition was induced in rats by increasing litter size to 18 pups per dam from birth until weaning. These offspring received a $22 \%$ protein diet from days $21-90$ of life. Control dams nursed 6 pups, which were fed the normal diet after weaning; all animals were killed at 90 days. Uterine estradiol receptors were measured according to the technique of Feherty $e t$ al., recovering bound estradiol with DEAE-cellulose filters instead of dextran-charcoal. Estradiol binding capacity of the uteri of marasmic rats was decreased compared to normal, age-matched controls expressed as picomoles per mg tissue $\left(8.26 \pm 2.76 \times 10^{-4}\right.$ vs. $5.16 \pm 1.96 \times 10^{-4}$, respectively, $\left.P<0.025\right)$, picomoles per $\mathrm{mg}$ protein $(0.262 \pm 0.0055$ vs. $0.0153 \pm 0.0054$, respectively, $P<$ $0.001)$, or picomoles per DNA $\left(1.96 \pm 0.80 \times 10^{-4}\right.$ vs. $1.10 \pm 0.28$ $\times 10^{-4}$, respectively, $P<0.025$ ). These results show that the number of estradiol receptors in the uterus of rats malnourished early in life is decreased. This is more evidence that early malnutrition produces damage in the uterus of the rat.

\section{Bioavailability of Heme-Iron Preparations Derived from Bovine Red Blood Cells}

E. HERTRAMPF, M. AMAR, AND A. STEKEL Institute of Nutrition and Food Technology, Universidad de Chile, Chile

In the past, milk has been fortified with inorganic iron compounds. The bioavailability of these compounds for absorption is altered according to the properties of the vehicle. The purpose of this work was to study the possibility of fortifying whole milk with organic iron obtained from fresh bovine red blood cells. The organic iron preparations used in this study were: 1) hemoglobin with stroma, 2) hemoglobin without stroma, 3) hemin. These compounds are soluble in milk and water (with the exception of hemin), do not alter the flavor of the milk, and give the milk a chocolate color. The absorption of these preparations was studied using both whole milk and aqueous solutions as vehicles by a double isotope technique. Seventy healthy infants from 6-18 months old were distributed into five groups: 1) hemoglobin with stroma in whole milk, 2) hemoglobin without stroma in whole milk, 3) hemin in whole milk, 4) hemoglobin with stroma in water, 5) hemin in water.

On day 1 each group was given $150 \mathrm{ml}$ milk or water to which were added $2 \mathrm{mg} \mathrm{Fe}$ in the form of the heme-iron being studied (hemoglobin with stroma, hemoglobin without stroma, or hemin) and the same product prepared from calf red blood cells that were biologically marked with ${ }^{55} \mathrm{Fe}(0.5 \mu \mathrm{Ci} / \mathrm{kg})$. On day 2 , the infants received $50 \mathrm{ml}$ of a ferrous-ascorbate solution that contained $2 \mathrm{mg}$ iron $\left(\mathrm{FeSO}_{4}\right)$ marked with ${ }^{59} \mathrm{Fe}(0.1 \mu \mathrm{Ci} / \mathrm{kg})$. Absorption was calculated 14 days later according to circulating radioactivity. The heme-iron preparations in milk showed a geometric mean absorption of $20 \%$. The ratios among the formulas of day 1 in milk and day 2 did not show statistically significant differences. No difference between the absorption of hemoglobin in milk or in aqueous solution was observed; however, the hemin showed a lower absorption value $(P<0.01)$ in aqueous solution. It can be concluded that fortification of whole milk with hemoglobin preparations is feasible. The addition of hemoglobin preparations to milk appears to be an excellent combination because of the organoleptic characteristics and high bioavailability of iron.

\section{Study of the La Brosse Spot Test for 3-Methoxy-4-hydrox- ymandelic Acid (VMA)}

MARTA BARONTINI DE G. MOYANO, GLORIA M. LEVIN, AND C. BERGADA Division of Endocrinology, Hospital de Ninos, Buenos Aires, Argentina

The object of this paper is to compare the La Brosse test with the quantitative urinary VMA measurement (Pisano's technique). Substances whose breakdown products appear in the urine and give false positive results were eliminated from the diet. Seven hundred 24-hr urine samples belonging to 580 patients were studied. Fifty-six patients had sympathoblastomas, 15 pheochromocytomas; the remaining several pathologies were not related to a catecholamine increase. Four hundred twelve negative, 132 intermediate, and 156 positive reactions were obtained. The negative tests had normal VMA in all cases except in five urine samples (false negative) belonging to three patients with pheochromocytoma who had increased VMA (between 15.4 and 38.6 $\mathrm{mg} / 24 \mathrm{hr}$ ). The intermediate results had normal VMA in all cases except one patient with pheochromocytoma (VMA $18.4 \mathrm{mg} / 24$ hr). Among the positive tests, 70 showed increased VMA and 86 normal VMA (false positive FP). The former results belonged to 55 samples from sympathoblastoma patients and to 15 from pheochromocytoma patients. The FP occurred in 15 patients treated for sympathoblastoma and in 71 patients without any pathology associated with a catecholamine increase. Of the sympathoblastoma patients, 30 were studied before any treatment (group I) and 26 after treatment (group II). In group I, all but 1 yielded positive tests: 28 had increased VMA and 2 had normal VMA. From this group, 16 patients were followed during treatment: 11 continued with positive tests and increased VMA excretions and 5 converted to negative tests and normal VMA. In group II, 19 were negative, 4 were intermediate, and 3 , all with normal VMA, were positive. Only one patient persisted with positive tests in the subsequent evaluations.

In conclusion, the La Brosse test is a practical screening test to detect and follow the course of treatment in patients with sympathoblastoma who initially had positive tests and increased VMA. All these results must be interpreted with caution because there are false positive results and a $15 \%$ of sympathoblastoma patients have normal VMA. The false negative results $(1.2 \%)$ were observed in $17 \%$ of pheochromocytoma patients. The intermediate reactions were associated with normal VMA excretions except in one pheochromocytoma patient.

\section{Pathology of "Uninvolved" Renal Parenchyma in Nephro- blastoma}

GUILLERMO E. GALLO Department of Pathology, Hospital de Ninos, Buenos Aires, Argentina

Two hundred twenty-five primary renal tumors in children were histologically examined; 191 of these were nephroblastomas $(\mathrm{Nbl})$ (Wilms' tumor). In 61, sufficient residual parenchyma was available for study. In a 5-month-old girl admitted with corticoidresistant nephrotic syndrome, an $\mathrm{Nbl}$ was found and a nephrectomy was performed. In the uninvolved renal tissue a severe diffuse glomerular mesangial sclerosis was present; it led to her death in renal failure 6 months after onset. In 12 other specimens nephroblastomatosis was present. The most common lesions were subcapsular metanophric hamartomata (either as discrete nodules or as diffuse involvement of the superficial cortex). They were frequently associated with nodular renal blastema and occasion- 